\title{
Application of Interior Digital Display in 3D Effect Drawings
}

\author{
Sirong Liu \\ Hubei University of Technology \\ Wuhan, China \\ sirong_liu@yeah.net
}

\begin{abstract}
-ever since the human beings created the first piece of work tool by consciousness, during the millions of years, the behavior of art design has always been in every step of the development course of human history. Along with the development of times and civilization, there are always new artistic expression methods to create art works more suitable for the development for the human society. The article starts from the historic origin of design expression, summarizes the development course of design, focuses on the key technologies for digital display by software, discusses on the key technologies of V-Ray and so on, and gets a conclusion.
\end{abstract}

Keywords: Interior Design, CG, Digital Art Design, V-Ray

\section{INTRODUCTION}

Since the middle of the 1990s, CG technology got widely used in construction and interior design. Its advantages made great impact on the traditional hand painted presentation. At the beginning of the 1990s, computer aided design started to be used in the construction field in China, but at that time, designers mainly use AUTO-CAD to draw plan and elevation drawings and construction drawings and at the scheme design stage, it was still mainly hand painted. With the development of related software, the advantages started to show, and the traditional hand painted presentations were greatly impacted. By the end of the $20^{\text {th }}$ century, such impact can be called fatal to domestic presentation field [1].The impact mainly includes the following: first, it has the functions that hand painting cannot realize: correct perspective, clear material display, closer to realistic situation. Meanwhile, it can be made to be animation to show the design idea more completely and thoroughly which enriches the expression of effect drawings. Secondly, in the information society, many commercial behaviors do not happen on the negotiation table, but are done through remote communication to give information and get feedback [2]. A computer supplies such conditions though which the drawing maker and the purchaser can communicate with each other with internet. Thirdly, compared with other methods, the biggest advantage of computer is that it is easy for modification. Reselection and recreation of color, form and material etc. can be made on the finished drawing. This is beneficial for the designer to optimize the design scheme to present the design idea from multiple aspects and to make the designer and the purchaser to express their own opinions and enhance cooperation to get ideal choice [3].

\section{APPLICATION OF MULTIMEDIA IN THE PRESENTATION OF DESIGN INFORMATION}

Multimedia programs are the application software made by multimedia technology. Such programs are very rich in contents with picture, text and sound and are very popular with wider range of application. The multi-dimension and multi-aspect presentation of space is more suitable for the needs and tendency of design, and with the maturity of technology, many families have got multimedia computer, which makes it necessary and possible for the application of multimedia presentation [4]. Digital display is to show the design scheme using multimedia technology through text, pictures, sound, and animation etc. The future environment results are shown completely even before the construction starts, which makes the customer feels he is there in person [5]. Its realization process includes situation analysis, overall structure conception, drafting, structure design, material collection and processing, software programming and testing etc. It can communicate with users through network. Multimedia presentation has both pictures and texts which shows the future environment artistically and completely. The application will be quite beneficial for the decoration companies to win bids. The digital design not only provides a new way for decoration design display but also provides new thinking for construction and garden design and other design displays.

People have multiple ways to receive information, and the two main methods for people receiving and spreading information are "by eyes" and "by ears". $65 \%$ of the information received by sense organs is from visual observation and $20 \%$ from sound, $10 \%$ from other sense of touch, $3 \%$ from sense of smell, and $2 \%$ from sense of taste. Visual and audio information has taken the biggest proportion [6]. Multimedia technology here is to combine the independent media like text, sound, picture, animation and video etc together to form a totally new media of display through computer software and hardware operations like information acquisition, operation, editing and storage etc. It changes the human machine interface, breaks the rigidity and complex of human machine dialogue, utilizes the processible information completely, mobilizes subjective initiative and makes the computer a non-professional tool fully utilized by people which makes work and life more convenient [7].

From the aspect of information processing, multimedia computer provides a completely new method for information display. It provides a digital processing method using computer technology to display, spread and process 
information complete with visual and audio information. The perfect combination of visible (text, picture, graph, animation etc.) and audible (language, music etc.) media realizes complete and natural information display and maximum information reception [8]. Thus it has more advantages than single method and static effect drawing and makes it more reasonable for people to explore the usage of multimedia to display design information.

\section{A COMPLETELY NEW INFORMATION SPREAD METHOD BROUGHT BY NETWORK}

Internet is the biggest computer digital communication network in the world, which is a global network composed of computers using common language for communication [9]. Internet combines network, multimedia and hyper text technologies and embodies the development tendency of combination of various information technologies. Its role is thus further displayed in the network environment. Meanwhile, it provides new network operation platform for teaching, research, commercial advertisement, remote medicine and metrological forecasts etc. From 1994, we have set up CSTNET, CERNER, CHINANET and CHINAGBR and the basic layout with these 4 networks is formed. INTERNET has not got a long history in China but has a surprisingly fast development speed with website localization as focus [10]. Currently, there are more and more Chinese websites and the information in Chinese on the websites are thus enriched. At the same time, the number of network proxy service enterprises is increasing, and service to new and old customers is becoming more convenient. Therefore the number of internet users in China is increasing fast. The prices of computer and accessories are coming down greatly, the number of computers in middle income families is increasing, and "family online" is becoming common. It has become a new channel for families to obtain latest, complete and vivid information through internet. Socialized internet will become a fresh media that can bring great changes to life after television and telephone. It is coming inside life, study and work of people with an unprecedented speed. To the family internet users, network has realized education in family, work in family and computerized family life. With the increasing of individual user groups, more and more enterprises have realized that they should join in the internet, and the application of internet shall transfer from initial information distribution to E-business.

\section{EXCELLENT THIRD PARTY RENDERER V-RAY}

V-Ray is the renderer plug-in issued by Chaosgroup for ads-Max, developed by the CG lovers in East Europe. Though the development scale is not big, the render effect of V-Ray is not worse than the effect of other renderers developed by big companies. V-Ray has a whole set of reflection, refraction and light transmission solutions. It is good at showing the texture of metal and glass etc. and creates true caustic effect. And the light transmission speed of it is also fast. Thus it is widely used in industrial design and construction display. Due to its concise parameters and convenience for understanding, many designers prefer it

The light object is the emulated object of various types of light sources by ads-Max, including common light fittings in households and offices, lighting mechanics for movie scenes and even daylight. Different light sources produce different lighting methods, and different lighting objects are formed in ads-Max.

a) To increase lighting intensity of scenes. In default status, the lighting intensity is usually not enough and many complicated objects cannot be displayed in a good manner. Thus more light needs to be added to increase lighting intensity.

b) To improve the reality of scenes through vivid lighting effects.

c) To provide shade in the scenes to increase the level of reality.

d) To emulate the light source in the scenes. The light object itself cannot be rendered, thus geometric model needs to be set up to suit the light source. Materials with light emission can also facilitate a lot.

e) To create scenes with photometric web lighting effect. Various lighting distribution effects can be easily made through setting up various photometric web file for photometric light. These photometric web files can be obtained from the manufacturers directly.

The light sources supplied by ads-Max can be classified into standard, photometric and daylight types. They both appear as light object in the view and have many common parameters including formation of shade. V-Ray is much used in modern display industry. How to show the light-shade effect in reality using V-Ray? This needs to be started from light rays.

There are two types of light rays in nature, point light source, i.e. the light source is relatively far from the lighted object and the volume of the light source can be ignored. The other is volume light source, i.e. the light source is not that far from the lighted object, and the volume of the light source must be considered. The sun, filament lamp, focus lamp and reflector lamp belong to point light source. Daylight, fluorescent lamp, energy saving lamp and display screen belong to volume light source. If we observe it carefully, we will find that the shade formed by point light source is different from that by volume source in form. The shade formed by point light source is often sharp edged, e.g. the reflection of buildings under strong sunshine is often sharp. If we read in filament light for long, we will feel uncomfortable because the shade there is very strong. The spotlight on stage has good focus effect with also strong shade so as to highlight the actors and objects. And the volume light source usually forms gentle shade. For example. The shade of buildings is obscure in cloudy days and even invisible. We can read for long under fluorescent light or energy saving light. The hospital shadowless lamp adopts the same principle. Why are the shades by point light source and volume light source different? See below for the analysis using knowledge of physics. (See figure 1) 


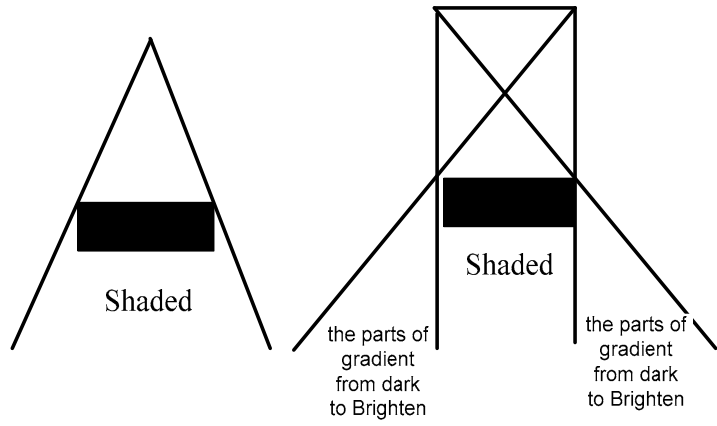

Figure 1. principle of shade formation by point light source and volume light source

The left figure is the shade schematic for point light source. We can see that the light rays from point light source has the object boundary as the boundary, which means the boundary line connecting the light point and the object boundary is the boundary line of the shade. And the shade is very sharp. The right figure is the shade schematic for volume light source. Here we show it using form similar to fluorescent lamp. We can view the light source as composed by numerous point light sources. Each light source emits lights around. Then we take the left and right poles of the volume light source as study object. Through analysis method similar to point light source, we can find that the shade formed by the rays from two poles is darkest in the middle and second darkest at the two sides. As there are numerous points, we can deduce that the shade of this volume light source under the same object should be: absolutely dark in the middle and lighter to the sides until there is totally no shade.

Thus we can deduce the following rules:

The shade of point light source is absolutely dark, and the shade of volume light source is obscure.

The larger the light emission area is on the volume light source, the more obscure its shade is.

The light focus effect of point light source is good. Emission distance is long and is suitable for local lighting. The diffuse effect of volume light source is good and the lighting distance is short suitable for overall lighting.

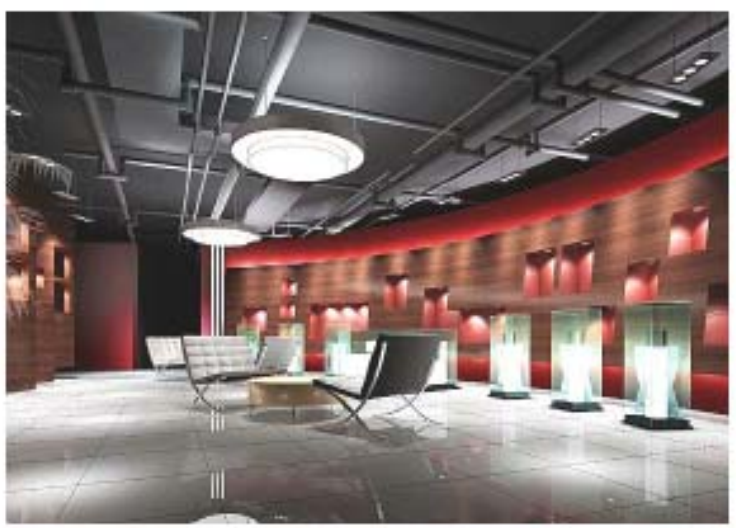

Figure 2. example of display effect of point light source

This effect drawing is mainly set up by point light source. We can see that the shade effect in this figure is relatively strong, and the important elements can be better displayed. Such a space with mainly point light source is quite artistic with clear importance level of the objects. The important parts are highlighted by point light source, and the other parts have no light or dark light. But to human living environment, such light distribution does not suit physical and psychological needs of human. The living space shall be mainly with volume light source to create a comfortable and gentle visual effect and elegant light-shade effect. Only the special needs of some places will adopt point light source.

\section{CONCLUSION}

It is convenient to use V-Ray for setting and the effect is easy to be controlled. The realistic effect is easier to be obtained. However, the render time will be relatively longer. Besides, the following differences shall be notices during the setting of linear scanner and V-Ray:

The setting of material reflection and refraction effect is different. V-Ray uses the reflection method of vraymap, while the linear scanner uses aytrace. In case of wrong setting, the result will be completely different. There will be even computer shutdown or restart.

The shade method of main light source is different. The shade of V-Ray shall be vrayshadow, and the shade of the main light source of linear scanner shall be aytraced shadow. No mistake shall be made here.

The role of background color is different. Background color in linear scanner is merely a color behind the main object without influence on the object color, whereas in V-Ray, the background color has influence on the object to make it reflect the background color. Thus when using V-Ray as the renderer, we should adjust the background color carefully to make it facilitate the lighting to the object.

\section{REFERENCE}

[1] B. Blundell and A. Schwarz, Volumetric Three- Dimensional Display Systems, John Wiley \& Sons, 2000.

[2] E.A. Downing et al., "A Three-Color, Solid-State, Three-Dimensional Display," Science, vol. 273, 1996, pp. 1185-1189.

[3] T. Honda et al., "Three-Dimensional Display Technologies Satisfying 'Super Multiview Condition,'” B. Javidi and F. Okano, eds., Proc. Three-Dimensional Video and Display: Devices And Systems, vol. CR76, SPIE Press, 2000, pp. 218-249.

[4] K. Langhans, et al., "New Portable FELIX 3D Display,"Proc. SPIE, vol. 3296, SPIE—Int'1 Soc. forOptical Eng., 1998, pp. 204-216.

[5] M. Halle, "Autostereoscopic Displays and Computer Graphics," Computer Graphics, ACM Siggraph, vol. 31, no. 2, 1997, pp. 58-62.

[6] G.E. Favalora et al., "100 Million-Voxel Volumetric Display," Cockpit Displays IX: Displays for Defense Applications, Proc. SPIE, vol. 4712, SPIE-Int'l Soc. for Optical Eng., 2002, pp. 300-312.

[7] W.-S. Chun et al., "Spatial 3D Infrastructure: Display- Independent Software Framework, High-Speed Rendering Electronics, and Several New Displays," Proc. SPIE-IS\&T Electronic Imaging, vol. 5664, 2005, pp. 302-312.

[8] T. Grossman, D. Wigdor, and R. Balakrishnan, "Multi-Finger Gestural Interaction with 3D Volumetric Displays," Proc. ACM Symp. User Interface Software and Technology, ACM Press, 2004, pp. 61- 70 .

[9] Shmuel Nitzan, Jacob Paroush. Optimal Decision Rules in Uncertain Dichotomous Choice Situations. International Economic Review. 
1982, 23 (2): 289-297P

[10] Richard E. Wendell. Multiple Objective Mathematical Programming with Respect to Interior Design . Operations Research. 1980

Published by Atlantis Press, Paris, France.

(C) the authors 